Wen-Yi Li and Guang-Chuan Ou*

\title{
Crystal structure of $\left[\left(\mu_{2}\right.\right.$-succinato $\left.\mathrm{k}^{3} 0,0^{\prime}: 0^{\prime \prime}\right)$-bis- $(5,5,7,12,12,14$-hexamethyl- 1,4,8,11-tetraazacyclotetradecane)]dinickel(II)] diperchlorate, dihydrate $\mathrm{C}_{36} \mathrm{H}_{82} \mathrm{Cl}_{2} \mathrm{~N}_{8} \mathrm{Ni}_{2} \mathrm{O}_{15}$
}

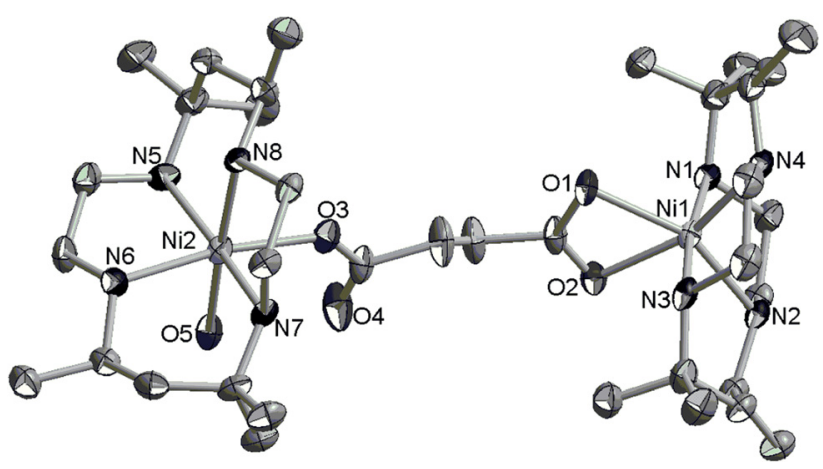

https://doi.org/10.1515/ncrs-2020-0616

Received December 1, 2020; accepted January 8, 2021; published online January 22, 2021

\begin{abstract}
$\mathrm{C}_{36} \mathrm{H}_{82} \mathrm{Cl}_{2} \mathrm{~N}_{8} \mathrm{Ni}_{2} \mathrm{O}_{15}$, monoclinic, $P 2_{1} / n$ (no. 14), $a=17.657(3) \AA, \quad b=16.047(3) \AA, c=18.346(3) \AA$, $\beta=109.062(4)^{\circ}, V=4913.2(16) \AA^{3}, Z=4, R_{g t}(F)=0.0561$, $w R_{\text {ref }}\left(F^{2}\right)=0.1664, T=173(2) \mathrm{K}$.
\end{abstract}

CCDC no.: 2054632

The molecular structure is shown in the figure (couter anions and the water molecules are omitted for clarity). Table 1 contains crystallographic data and Table 2 contains the list of the atoms including atomic coordinates and displacement parameters.

\section{Source of material}

An acetonitrile solution $(20 \mathrm{~mL})$ of $[\mathrm{NiL}]\left(\mathrm{ClO}_{4}\right)_{2}(0.54 \mathrm{~g}$, $1 \mathrm{mmol})(\mathrm{L}=5,5,7,12,12,14$-hexamethyl-1,4,8,11-tetra-

*Corresponding author: Guang-Chuan Ou, Department of Biology and Chemistry, Hunan University of Science and Engineering, Yongzhou, Hunan 425199, P. R. China, E-mail: ogcouguangchuan@163.com. https://orcid.org/0000-0002-1005-8519

Wen-Yi Li, Key Laboratory of Functional Metal-Organic Compounds of Hunan Province, College of Chemistry and Materials Science, Hengyang Normal University, HengYang, Hunan 421008, P. R. China, E-mail:757189515@qq.com
Table 1: Data collection and handling.

\begin{tabular}{ll}
\hline Crystal: & Blue block \\
Size: & $0.41 \times 0.40 \times 0.21 \mathrm{~mm}$ \\
Wavelength: & Mo $K \alpha$ radiation $(0.71073 \AA)$ \\
$\mu:$ & $0.95 \mathrm{~mm}^{-1}$ \\
Diffractometer, scan mode: & $\varphi$ and $\omega$ \\
$\theta_{\text {max }}$, completeness: & $27.0^{\circ}, 99 \%$ \\
$N\left(h k l_{\text {measured }}, N(h k l)_{\text {unique }}, R_{\text {int }}:\right.$ & $21,783,10,493,0.033$ \\
Criterion for $I_{\text {obs }}, N(h k l)_{\text {gt }}:$ & $I_{\text {obs }}>2 \sigma\left(I_{\text {obs }}\right), 6805$ \\
$N(\text { param })_{\text {refined }}:$ & 598 \\
Programs: & Bruker [1], SHELX [2, 3], Diamond [4]
\end{tabular}

azacyclotetradecane) was added to a mixed solution of succinic acid (0.06 g, $0.5 \mathrm{mmol})$ and $\mathrm{NaOH}(0.04 \mathrm{~g}, 1 \mathrm{mmol})$ in water $(10 \mathrm{~mL})$. The resulting blue solution was evaporated slowly at room temperature to give blue prism-shaped crystals for several days.

\section{Experimental details}

The structure was solved using direct methods, which yielded the positions of all non-hydrogen atoms. All hydrogen atoms of the ligands were placed in calculated positions with fixed isotropic thermal parameters and included in the structure factor calculations in the final stage of refinement. The $U_{\text {iso }}$ values of the hydrogen atoms of methyl groups were set to $1.5 U_{\text {eq }}(\mathrm{C})$ and the $U_{\text {iso }}$ values of all other hydrogen atoms were set to $1.2 U_{\text {eq }}(\mathrm{C}, \mathrm{N})$.

\section{Comment}

The design and synthesis of extended bridged polymetallic complexes have attracted much interest in the development of materials chemistry. Some complexes bridged by dicarboxylato ligands have been reported [5-6].

$\mathrm{X}$-ray crystal structural analysis reveals that the asymmetric unit of the title structure contains one 
Table 2: Fractional atomic coordinates and isotropic or equivalent isotropic displacement parameters $\left(\AA^{2}\right)$.

\begin{tabular}{|c|c|c|}
\hline Atom & $x$ & $y$ \\
\hline C1 & $0.7542(2)$ & 1.1454 (3) \\
\hline $\mathrm{H} 1 \mathrm{~A}$ & 0.7322 & 1.2012 \\
\hline H1B & 0.8044 & 1.1377 \\
\hline $\mathrm{C} 2$ & $0.7709(2)$ & $1.1393(3)$ \\
\hline $\mathrm{H} 2 \mathrm{~A}$ & 0.7933 & 1.0837 \\
\hline $\mathrm{H} 2 \mathrm{~B}$ & 0.8108 & 1.1820 \\
\hline $\mathrm{C} 3$ & $0.7042(2)$ & $1.1324(2)$ \\
\hline H3 & 0.7133 & 1.0711 \\
\hline $\mathrm{C} 4$ & $0.7750(2)$ & $1.1776(3)$ \\
\hline $\mathrm{H} 4 \mathrm{~A}$ & 0.8245 & 1.1599 \\
\hline H4B & 0.7780 & 1.1643 \\
\hline $\mathrm{H} 4 \mathrm{C}$ & 0.7681 & 1.2379 \\
\hline $\mathrm{C} 5$ & $0.6273(2)$ & 1.1550 \\
\hline $\mathrm{H} 5 \mathrm{~A}$ & 0.6108 & 1.2114 \\
\hline H5B & 0.6406 & 1.1588 \\
\hline $\mathrm{C} 6$ & $0.5542(2)$ & 1.0967 (3) \\
\hline $\mathrm{C} 7$ & $0.5747(3)$ & 1.0084 (3) \\
\hline $\mathrm{H} 7 \mathrm{~A}$ & 0.5264 & 0.9736 \\
\hline H7B & 0.5951 & 1.0088 \\
\hline $\mathrm{H} 7 \mathrm{C}$ & 0.6157 & 0.9856 \\
\hline $\mathrm{C} 8$ & $0.4884(3)$ & 1.1289 (3) \\
\hline $\mathrm{H} 8 \mathrm{~A}$ & 0.4778 & 1.1878 \\
\hline $\mathrm{H} 8 \mathrm{~B}$ & 0.5063 & 1.1228 \\
\hline $\mathrm{H} 8 \mathrm{C}$ & 0.4392 & 1.0965 \\
\hline C9 & $0.4784(2)$ & 1.1679 (3) \\
\hline $\mathrm{H} 9 \mathrm{~A}$ & 0.5082 & 1.2195 \\
\hline $\mathrm{H} 9 \mathrm{~B}$ & 0.4274 & 1.1687 \\
\hline C10 & $0.4612(2)$ & $1.1645(3)$ \\
\hline $\mathrm{H} 10 \mathrm{~A}$ & 0.4307 & 1.1133 \\
\hline $\mathrm{H} 10 \mathrm{~B}$ & 0.4283 & 1.2132 \\
\hline C11 & $0.5265(2)$ & $1.1478(3)$ \\
\hline H11 & 0.5069 & 1.0892 \\
\hline C12 & 0.4648 & $1.2055(3)$ \\
\hline $\mathrm{H} 12 \mathrm{~A}$ & 0.4127 & 1.1976 \\
\hline H12B & 0.4601 & 1.1923 \\
\hline $\mathrm{H} 12 \mathrm{C}$ & 0.4819 & 1.2635 \\
\hline C13 & $0.6062(3)$ & $1.1550(3)$ \\
\hline $\mathrm{H} 13 \mathrm{~A}$ & 0.6321 & 1.2075 \\
\hline H13B & 0.5942 & 1.1606 \\
\hline C14 & $0.6680(3)$ & $1.0845(3)$ \\
\hline C15 & $0.7382(3)$ & $1.1017(3)$ \\
\hline $\mathrm{H} 15 \mathrm{~A}$ & 0.7576 & 1.1587 \\
\hline $\mathrm{H} 15 \mathrm{~B}$ & 0.7204 & 1.0952 \\
\hline $\mathrm{H} 15 \mathrm{C}$ & 0.7816 & 1.0622 \\
\hline C16 & 0.6318 & $1.0004(3)$ \\
\hline H16A & 0.6735 & 0.9574 \\
\hline H16B & 0.6094 & 1.0028 \\
\hline $\mathrm{H} 16 \mathrm{C}$ & 0.5892 & 0.9867 \\
\hline C17 & $0.6036(2)$ & $0.9251(2)$ \\
\hline $\mathrm{C} 18$ & $0.5965(3)$ & $0.8326(3)$ \\
\hline $\mathrm{H} 18 \mathrm{~A}$ & 0.6068 & 0.8036 \\
\hline $\mathrm{H} 18 \mathrm{~B}$ & 0.6379 & 0.8145 \\
\hline C19 & $0.5172(3)$ & $0.8081(3)$ \\
\hline
\end{tabular}

Table 2: (continued)

\begin{tabular}{|c|c|c|c|c|}
\hline Atom & $x$ & $y$ & $z$ & $U_{\text {iso }}{ }^{*} / U_{\text {eq }}$ \\
\hline H19A & 0.4780 & 0.8142 & 0.1996 & $0.063^{*}$ \\
\hline H19B & 0.5015 & 0.8476 & 0.2861 & $0.063^{*}$ \\
\hline $\mathrm{C} 20$ & $0.5105(2)$ & $0.7197(2)$ & $0.2801(3)$ & $0.0328(9)$ \\
\hline $\mathrm{C} 21$ & $0.2564(2)$ & 0.6489 (3) & $0.2818(2)$ & $0.0315(9)$ \\
\hline $\mathrm{H} 21 \mathrm{~A}$ & 0.2289 & 0.6942 & 0.3000 & $0.038^{*}$ \\
\hline $\mathrm{H} 21 \mathrm{~B}$ & 0.2267 & 0.5965 & 0.2812 & $0.038^{*}$ \\
\hline $\mathrm{C} 22$ & $0.2570(2)$ & $0.6684(2)$ & $0.2017(2)$ & $0.0311(9)$ \\
\hline $\mathrm{H} 22 \mathrm{~A}$ & 0.2014 & 0.6751 & 0.1664 & $0.037^{\star}$ \\
\hline $\mathrm{H} 22 \mathrm{~B}$ & 0.2862 & 0.7211 & 0.2021 & $0.037^{\star}$ \\
\hline $\mathrm{C} 23$ & $0.3116(3)$ & $0.6204(3)$ & $0.1012(2)$ & $0.0341(9)$ \\
\hline $\mathrm{H} 23$ & 0.3518 & 0.6666 & 0.1120 & 0.041 * \\
\hline $\mathrm{C} 24$ & $0.2354(3)$ & $0.6497(3)$ & $0.0382(3)$ & 0.0475 (12) \\
\hline $\mathrm{H} 24 \mathrm{~A}$ & 0.1934 & 0.6075 & 0.0305 & $0.071^{*}$ \\
\hline $\mathrm{H} 24 \mathrm{~B}$ & 0.2469 & 0.6580 & -0.0101 & $0.071^{*}$ \\
\hline $\mathrm{H} 24 \mathrm{C}$ & 0.2172 & 0.7024 & 0.0539 & $0.071^{*}$ \\
\hline $\mathrm{C} 25$ & $0.3469(2)$ & $0.5444(3)$ & $0.0727(2)$ & $0.0343(9)$ \\
\hline $\mathrm{H} 25 \mathrm{~A}$ & 0.3121 & 0.4961 & 0.0726 & $0.041^{*}$ \\
\hline $\mathrm{H} 25 \mathrm{~B}$ & 0.3428 & 0.5551 & 0.0184 & $0.041^{*}$ \\
\hline $\mathrm{C} 26$ & $0.4335(2)$ & $0.5184(3)$ & $0.1160(2)$ & $0.0341(10)$ \\
\hline $\mathrm{C} 27$ & $0.4555(3)$ & $0.4461(3)$ & $0.0716(3)$ & 0.0441 (11) \\
\hline $\mathrm{H} 27 \mathrm{~A}$ & 0.5100 & 0.4269 & 0.0996 & $0.066^{*}$ \\
\hline $\mathrm{H} 27 \mathrm{~B}$ & 0.4528 & 0.4651 & 0.0201 & $0.066^{*}$ \\
\hline $\mathrm{H} 27 \mathrm{C}$ & 0.4177 & 0.4000 & 0.0670 & $0.066^{*}$ \\
\hline C28 & $0.4921(3)$ & $0.5903(3)$ & $0.1197(3)$ & 0.0460 (12) \\
\hline $\mathrm{H} 28 \mathrm{~A}$ & 0.4834 & 0.6346 & 0.1528 & $0.069^{*}$ \\
\hline $\mathrm{H} 28 \mathrm{~B}$ & 0.4830 & 0.6122 & 0.0677 & 0.069 * \\
\hline $\mathrm{H} 28 \mathrm{C}$ & 0.5472 & 0.5698 & 0.1409 & $0.069^{\star}$ \\
\hline $\mathrm{C} 29$ & $0.4076(3)$ & $0.4100(2)$ & $0.2033(3)$ & $0.0358(10)$ \\
\hline $\mathrm{H} 29 \mathrm{~A}$ & 0.4385 & 0.3661 & 0.1875 & $0.043^{\star}$ \\
\hline H29B & 0.3518 & 0.4081 & 0.1675 & $0.043^{*}$ \\
\hline C30 & $0.4078(3)$ & $0.3924(2)$ & $0.2834(3)$ & $0.0351(10)$ \\
\hline $\mathrm{H} 30 \mathrm{~A}$ & 0.3813 & 0.3382 & 0.2844 & $0.042^{*}$ \\
\hline H30B & 0.4637 & 0.3888 & 0.3187 & $0.042^{*}$ \\
\hline C31 & $0.3774(2)$ & $0.4557(3)$ & $0.3943(2)$ & $0.0327(9)$ \\
\hline H31 & 0.4348 & 0.4692 & 0.4226 & 0.039 * \\
\hline C32 & $0.3596(3)$ & $0.3684(3)$ & $0.4197(3)$ & $0.0422(11)$ \\
\hline H32A & 0.3035 & 0.3540 & 0.3925 & $0.063^{*}$ \\
\hline H32B & 0.3693 & 0.3683 & 0.4754 & $0.063^{*}$ \\
\hline $\mathrm{H} 32 \mathrm{C}$ & 0.3947 & 0.3275 & 0.4073 & $0.063^{\star}$ \\
\hline $\mathrm{C} 33$ & $0.3253(2)$ & $0.5203(3)$ & $0.4166(2)$ & $0.0346(10)$ \\
\hline $\mathrm{H} 33 \mathrm{~A}$ & 0.3258 & 0.5067 & 0.4694 & $0.042^{\star}$ \\
\hline H33B & 0.2696 & 0.5123 & 0.3819 & $0.042^{*}$ \\
\hline C34 & $0.3450(2)$ & $0.6132(3)$ & $0.4153(2)$ & $0.0310(9)$ \\
\hline C35 & $0.4294(2)$ & $0.6342(3)$ & $0.4677(2)$ & $0.0369(10)$ \\
\hline $\mathrm{H} 35 \mathrm{~A}$ & 0.4687 & 0.6066 & 0.4485 & $0.055^{*}$ \\
\hline H35B & 0.4364 & 0.6150 & 0.5202 & $0.055^{*}$ \\
\hline $\mathrm{H} 35 \mathrm{C}$ & 0.4375 & 0.6947 & 0.4681 & $0.055^{*}$ \\
\hline C36 & $0.2845(3)$ & $0.6628(3)$ & $0.4430(3)$ & 0.0417 (11) \\
\hline $\mathrm{H} 36 \mathrm{~A}$ & 0.2899 & 0.7224 & 0.4343 & $0.063^{*}$ \\
\hline H36B & 0.2952 & 0.6528 & 0.4982 & $0.063^{*}$ \\
\hline $\mathrm{H} 36 \mathrm{C}$ & 0.2299 & 0.6447 & 0.4142 & $0.063^{*}$ \\
\hline $\mathrm{Cl} 1$ & $0.66033(7)$ & $0.10390(7)$ & $0.66170(7)$ & $0.0425(3)$ \\
\hline $\mathrm{Cl} 2$ & $0.85128(6)$ & $0.88737(6)$ & $0.20413(7)$ & $0.0358(2)$ \\
\hline N1 & $0.69523(18)$ & 1.07966 (19) & $0.17206(18)$ & $0.0267(7)$ \\
\hline
\end{tabular}


Table 2: (continued)

\begin{tabular}{|c|c|c|c|c|}
\hline Atom & $x$ & $y$ & $z$ & $U_{\text {iso }} * / U_{\text {eq }}$ \\
\hline $\mathrm{H} 1 \mathrm{C}$ & 0.7250 & 1.0258 & 0.1855 & $0.032^{\star}$ \\
\hline N2 & $0.69522(17)$ & 1.15238 (19) & $0.31433(18)$ & $0.0256(7)$ \\
\hline $\mathrm{H} 2 \mathrm{C}$ & 0.6791 & 1.2122 & 0.3045 & 0.031 * \\
\hline N3 & $0.52669(18)$ & 1.09387 (19) & $0.2952(2)$ & $0.0295(8)$ \\
\hline $\mathrm{H} 3 \mathrm{~A}$ & 0.4896 & 1.0451 & 0.2802 & $0.035^{*}$ \\
\hline N4 & $0.53820(18)$ & 1.16500 (19) & 0.15537 (19) & $0.0290(7)$ \\
\hline $\mathrm{H} 4 \mathrm{D}$ & 0.5628 & 1.2215 & 0.1682 & $0.035^{*}$ \\
\hline N5 & $0.4429(2)$ & $0.4926(2)$ & 0.19741 (19) & $0.0318(8)$ \\
\hline $\mathrm{H} 5 \mathrm{C}$ & 0.5018 & 0.4864 & 0.2239 & $0.038^{\star}$ \\
\hline N6 & 0.36456 (19) & $0.4598(2)$ & $0.30986(19)$ & $0.0286(7)$ \\
\hline $\mathrm{H} 6 \mathrm{~A}$ & 0.3059 & 0.4527 & 0.2821 & $0.034^{\star}$ \\
\hline N7 & 0.33957 (18) & $0.6403(2)$ & $0.33500(18)$ & $0.0264(7)$ \\
\hline H7D & 0.3627 & 0.6978 & 0.3409 & 0.032 * \\
\hline N8 & $0.29694(18)$ & 0.59898 (19) & $0.17466(18)$ & $0.0256(7)$ \\
\hline $\mathrm{H} 8 \mathrm{D}$ & 0.2609 & 0.5493 & 0.1650 & $0.031^{*}$ \\
\hline $\mathrm{Ni1}$ & $0.61140(3)$ & $1.07906(3)$ & $0.23348(3)$ & $0.02325(14)$ \\
\hline $\mathrm{Ni} 2$ & 0.40119 & $0.57213(3)$ & $0.27082(3)$ & 0.02559 (14) \\
\hline 01 & $0.54938(16)$ & $0.96458(16)$ & 0.18808 (17) & $0.0322(6)$ \\
\hline $01 \mathrm{~W}$ & $0.8077(3)$ & $0.8876(4)$ & $0.3809(3)$ & 0.0969 (17) \\
\hline H1WA & 0.767 (3) & $0.920(4)$ & $0.362(4)$ & $0.116^{*}$ \\
\hline H1WB & $0.837(3)$ & $0.893(4)$ & 0.351 & $0.116^{*}$ \\
\hline 02 & $0.66254(16)$ & $0.96475(16)$ & $0.28406(16)$ & $0.0315(6)$ \\
\hline $02 \mathrm{~W}$ & 0.3829 & $0.9645(4)$ & 0.1474 & $0.134(2)$ \\
\hline $\mathrm{H} 2 \mathrm{WA}$ & $0.4340(13)$ & $0.955(6)$ & $0.160(5)$ & $0.161^{*}$ \\
\hline H2WB & $0.367(4)$ & $0.943(3)$ & $0.1828(17)$ & $0.161^{*}$ \\
\hline 03 & $0.44240(16)$ & $0.68719(17)$ & $0.25149(16)$ & $0.0336(7)$ \\
\hline 04 & 0.57152 (19) & $0.6872(2)$ & $0.3263(2)$ & $0.0556(9)$ \\
\hline 05 & 0.51771 (17) & $0.55019(17)$ & $0.35857(18)$ & $0.0351(7)$ \\
\hline $\mathrm{H} 5 \mathrm{D}$ & $0.532(2)$ & 0.5985 (11) & $0.346(2)$ & $0.042^{*}$ \\
\hline $\mathrm{H} 5 \mathrm{E}$ & $0.550(2)$ & $0.5133(15)$ & $0.353(3)$ & $0.042^{*}$ \\
\hline 06 & $0.5923(2)$ & $0.1546(2)$ & $0.6249(2)$ & 0.0557 (9) \\
\hline 07 & $0.7168(3)$ & $0.1465(3)$ & $0.7165(4)$ & $0.148(3)$ \\
\hline 08 & $0.6371(4)$ & $0.0375(3)$ & $0.7043(4)$ & $0.127(2)$ \\
\hline 09 & $0.6868(3)$ & $0.0595(3)$ & $0.6106(3)$ & 0.1096 (19) \\
\hline 010 & $0.7704(2)$ & $0.8593(2)$ & $0.1762(2)$ & $0.0635(10)$ \\
\hline 011 & $0.8563(2)$ & $0.9680(2)$ & $0.2372(2)$ & $0.0547(9)$ \\
\hline 012 & $0.8969(2)$ & $0.8313(2)$ & $0.2616(2)$ & 0.0766 (13) \\
\hline 013 & $0.8842(3)$ & $0.8919(3)$ & $0.1425(2)$ & 0.0791 (13) \\
\hline
\end{tabular}

$\left[(\mathrm{NiL})_{2}\left(\mathrm{C}_{4} \mathrm{H}_{4} \mathrm{O}_{4}\right)\left(\mathrm{H}_{2} \mathrm{O}\right)\right]^{2+}$ cation, two $\left[\mathrm{ClO}_{4}\right]^{-}$anions and two water molecules.

In contrast to similar structures [5-6], each $\mathrm{Ni}(\mathrm{II})$ atom displays a six-coordinate octahedral coordination geometry by coordination with four nitrogen atoms from macrocyclic ligand in a folded conformation, plus three oxygen atoms from the succinato ligand (see the figure). The $\mathrm{Ni}-\mathrm{N}$ bond lengths $[2.084(3)-2.164(3) \AA]$ are close to the $\mathrm{Ni}-\mathrm{O}$ bond lengths $[2.057(3)-2.187(3) \AA$ ] .

Author contributions: All the authors have accepted responsibility for the entire content of this submitted manuscript and approved submission.

Research funding: The NSFC (22002037), the Scientific Research Hunan Provincial Education Department (19A068), the Foundation of Key Laboratory of Functional MetalOrganic Compounds of Hunan Province (M019K03) and the Foundation of Hengyang Normal University (19Q015).

Conflict of interest statement: The authors declare no conflicts of interest regarding this article.

\section{References}

1. Bruker. APEX3, SAINT-Plus, XPREP; Bruker AXS Inc.: Madison, Wisconsin, USA, 2016.

2. Sheldrick G. M. SHELXT - Integrated space-group and crystalstructure determination. Acta Crystallogr. 2015, A71, 3-8.

3. Sheldrick G. M. Crystal structure refinement with SHELXL. Acta Crystallogr. 2015, C71, 3-8.

4. Brandenburg K. DIAMOND. Visual Crystal Structure Information System (ver. 4.0); Crystal Impact: Bonn, Germany, 2015.

5. Jiang L., Feng X. L., Lu T. B. Supramolecular architectures of macrocyclic nickel(II) complexes with defined structures of cis and trans configurations. Cryst. Growth Des. 2005, 5, 1469-1475.

6. Ou G. C., Yuan X. Y., Li Z. Z. Synthesis and crystal structure of two succinato-bridged macrocyclic nickel(II) complexes. Z. Anorg. Allg. Chem. 2013, 639, 158-162. 